\title{
Physics in Anaesthesia
}

\section{Ben Middleton, Justin Phillips, Rik Thomas, Simon Stacey. (CScion Publishing Ltd, Oxfordshire, UK, 2012. Trade paperback 352 pages. ISBN 9781904842989}

\author{
Michael E. Bourke, MD
}

Received: 4 September 2012/ Accepted: 7 September 2012/Published online: 19 September 2012

(c) Canadian Anesthesiologists' Society 2012

This textbook in trade paperback format - yes, I actually read it all- is designed as a teaching tool for anesthesia physicians in training, including a broader audience of medical, nursing, and operating room personnel. Although comprehensive, the text is beneficial and practical (intended) for those who consider themselves "non-physicists". The authors have diverse backgrounds, including a consultant anesthetist, anesthesia specialist trainee, biomedical engineer, and chief perfusionist; the latter two authors have physics backgrounds. To address the Fellowship of the Royal College of Anaesthetists (FRCA) training guidelines (UK), the text includes links to the contents guide and exam preparation and to the latest FRCA syllabus (www.scionpub lishing.com/physicsinanaesthesia $\rightarrow$ Resources).

This is an excellent refreshing and practical text when compared with various older textbooks on physics for anesthesia. The focus of its design and character is not only on the subject of physics, as emphasis is also placed on the process of learning itself. Classical topics are simplified and revised with current information; more recent topics are added or updated, and most elements of physics relating to anesthesia and medicine in general are addressed.

Each of the 29 concise chapters starts with a list of key elements that should be understood upon completion of that section. The text is written in a concise, uncomplicated, and easily understood manner, and representative clinical scenarios are often used. Diagrams and tables are mostly straightforward and well labelled; "must-learn" definitions are highlighted in separate outlined boxes; equations are clearly labelled; and "must-learn" equations

M. E. Bourke, MD ( $\square)$

University of Ottawa Heart Institute, Ottawa, ON, Canada

e-mail: mbourke@ottawaheart.ca are clearly noted. Learning is reinforced at the end of each chapter with a summary section that outlines the key points; and comprehension is further reinforced with a series of challenging single best answer and multiplechoice questions (the correct answers are included). An interested student cannot complete a chapter without knowing the essential material to learn.

The details in chapters 1-5 contain the usual basic information required: Atoms and matter (Ch 1), Simple mechanics (Ch 2), Energy and power (Ch 3), Temperature and heat (Ch 4), and Waves (Ch 5). The principles of harmonic analysis and Fourier transformation are mentioned, as are resonance and damping, although not as detailed as might be helpful in the "consultant" clinical setting. Pressure measurement (Ch 6), Humidity (Ch 7), and Measurement of gas flow (Ch 8) are relatively comprehensive chapters, but considering the prevailing importance of blood pressure measurement in the clinical setting, the description of noninvasive and invasive blood pressure monitoring could be expanded to allow a consultant to troubleshoot more effectively and compare techniques in the clinical setting. Principles of laminar flow, turbulent flow, Bernoulli's principle, the Venturi effect, and the Coanda effect are exceptionally well described. Gas laws (Ch 9), and Diffusion, osmosis and solubility (Ch 10) are described in a detailed but practical manner with the detail appropriate for an anesthetic consultant. Chapters 11-17 show the authors' current and previously published expertise in these areas: Measuring gas and vapour concentrations (Ch 11), Vaporizers (Ch 12), Medical gas supplies (Ch 13), Breathing systems and ventilation (Ch 14), Optics and oximetry (Ch 15), Blood flow measurement (Ch 16), and Equipment management (Ch 17). Subsequent chapters on Basics of electricity (UK standard), Electromagnetism and alternating current, 
Electrical shocks and safety, and Electrocardiography, pacing and defibrillation are comprehensive.

The topics in chapters 23-26 on Ultrasound, Lasers, Magnetic resonance imaging, and Nuclear physics and radiation are impressive; the principles are clear, and the concepts are up-to-date. For readers who are true nonphysicists, non-mathematicians, and less oriented with computers, chapters $22,27,28$, and 29 provide a concise overview of the useful aspects of monitors/computers, basic mathematics, and basic statistics (excellent).
In my view, this is a superb teaching textbook on basic physics. The North American audience will need to adapt pressure units (e.g., $\mathrm{kPa}$ to $\mathrm{mmHg}$ ) and electrical standards, but this is a minor issue relative to the overall content. I would recommend this textbook to our Anesthesia PostGraduate Program!

Competing interests None declared. 